\title{
Trabajos Fin de Grado y su Contribución al Triángulo del Conocimiento en la Educación Superior. Estudio Valorativo
}

\author{
Final Degree Projects and their Contribution to the Triangle of \\ Knowledge in Higher Education. Valorative Study
}

\author{
Carmen Guillén-Díaz * \\ Francisco Javier Sanz-Trigueros \\ Universidad de Valladolid, España
}

\begin{abstract}
En el marco de la oferta de formación universitaria de profesionales docentes del Grado en Maestro(a) de Educación Primaria, Menciones de Lengua Extranjera (Francés-Inglés), los autores centran la atención en la asignatura Trabajo Fin de Grado (TFG). Los objetivos de esta investigación son analizar la orientación a la investigación de los TFGs y caracterizarlos por la calidad de orientación a la investigación de sus elementos configuradores. Para ello se utiliza una muestra documental de TFG disponible, seleccionada de los años 2011-2017. Tomados dichos componentes como sistema categorial, y para un análisis cualitativo e interpretativo de las informaciones textuales, se aplica la técnica global del análisis de contenido. El corpus de datos obtenido según un tratamiento semántico manual y la presentación de resultados se organizan en torno a las tres determinaciones que, para los expertos, subyacen a la concepción y elaboración de estos trabajos. En las conclusiones, los puntos fuertes y obstáculos caracterizan su potencial como un espacio visible de investigación dinámica y estimulante, rico en expectativas para la mejora de la práctica educativa, contribuyendo a la excelencia en el desarrollo de la carrera profesional de estos docentes.
\end{abstract}

Descriptores: Análisis cualitativo; Competencias del docente; Documento primario; Formación de docentes de primaria; Investigación sobre la educación.

This article frames on the University training provision of teaching professionals of the Primary Education Degree, Mentions in Foreign Language (French-English). The authors draw the attention to the so-called subject Final Degree Project. The aims of this research are to analyse the research orientation of the projects and characterize them by the quality of research orientation of their elements. For this, a documentary sample of available final degree projects is used, selected from the years 2011-2017. The presence of consubstantial components of all research, taken as a categorical system for a qualitative and interpretive analysis, is studied in an available documentary sample selected from the years 2011-2017. Since the global technique of content analysis is implemented, both the corpus of data obtained from a manual processing and the presentation of the results, are organized around three determinations underlying the conception and elaboration of these projects. In the conclusions, the strengths and drawbacks characterize the potential of Final Degree Projects as a visible place for dynamic and stimulating research, rich in expectations for the improvement of educational practice. This enables contributing to excellence in the development of the professional career of these teachers.

Keywords: Qualitative analysis; Teacher qualifications; Primary documents; Primary teacher training; Educational research.

*Contacto: ccmc@dlyl.uva.es

ISSN: $1696-4713$

www.rinace.net/reice/

revistas.uam.es/reice
Recibido: $\quad 12$ de diciembre 2018

$1^{\text {a }}$ Evaluación: 3 de enero 2019

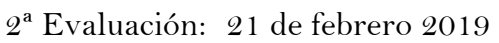

Aceptado: $\quad 11$ de marzo 2019 


\section{Introducción}

Ante el Comunicado emitido el 25 de mayo de 2018 en París para celebrar la creación del Espacio Europeo de Educación Superior, coincidiendo con los 20 años transcurridos desde la firma de la Declaración de la Sorbona, y al mismo tiempo, apenas cumplidas dos décadas de la Conferencia Mundial sobre la Educación Superior (París, 5 al 9 de octubre de 1998), resulta ineludible acercarnos a los documentos aprobados por sus participantes. Se trata de la "Declaración Mundial sobre la Educación Superior en el siglo XXI: Visión y acción y del Marco de Acción prioritaria para el cambio y el desarrollo de la Educación Superior" (UNESCO, 1998).

En ellos, se promueve una estrategia de compromiso por asegurar la calidad de la Educación Superior y el desarrollo de políticas para afrontar retos y desafíos contemporáneos, tales como el reconocimiento de cualificaciones profesionales, el desarrollo profesional continuo y la calidad de la enseñanza.

Y es, en la citada Declaración Mundial (UNESCO, 1998), en donde ya encontramos el consenso sobre la pertinencia y mejora de la calidad de los Planes de estudios que están destinados a preparar a los profesores de los niveles preescolar, primario y secundario (Artículo 10). Lo cual, en este servicio público que constituye la Educación Superior (Artículo 14), atañe de forma particular a la oferta del Grado en Maestro(a) de Educación Primaria, Menciones de Lengua Extranjera (Francés-Inglés)ํ. Es un Grado universitario, destinado a la formación inicial de docentes especialistas, a quienes se confía el desarrollo de la competencia clave en comunicación lingüística ${ }^{2}$, conforme a la gran determinación de política lingüística educativa ${ }^{3}$ : una Educación plurilingüe e intercultural desde las primeras edades, para aumentar en los individuos sus posibilidades de movilidad y empleabilidad. Es por lo que el establecimiento curricular de la enseñanza y el aprendizaje de lenguas constituye un desafío y un compromiso comunes vinculados a la política de calidad en la Universidad española.

Ese consenso está presidido por la reafirmada necesidad que recoge el epígrafe correspondiente a Misiones y funciones de la Educación Superior, Artículo 1. La misión de educar, formar y realizar investigaciones ${ }^{4}$ :

\footnotetext{
formar diplomados altamente cualificados y ciudadanos responsables, capaces de atender a las necesidades de todos los aspectos de la actividad humana, ofreciéndoles cualificaciones que estén a la altura de los tiempos modernos (...) conocimientos teóricos y prácticos de alto nivel. (p. 21)
}

\footnotetext{
${ }^{1}$ Formación universitaria en la que los autores desempeñan sus funciones y tareas de docencia e investigación, en asignaturas adscritas y asignadas al área de conocimiento de Didáctica de la Lengua y la Literatura. Área del ámbito científico de las Ciencias Sociales, creada por el Consejo de Universidades a raíz de la Ley Orgánica 11/1983, de 25 de agosto, de Reforma Universitaria. (España), y en la que se encuentra integrado el ámbito disciplinar de la Didáctica de las Lenguas-Culturas Extranjeras. Esta denominación fue acuñada por Galisson (1990), dando cuenta de su vocación primera: la formación a la docencia y a la investigación y el desarrollo de la investigación.

${ }^{2}$ Actualizada ésta en el documento Comisión Europea (2018a), en términos de Competencia multilingüe.

${ }^{3}$ Una aproximación a su descripción -desde sus orígenes-, fue realizada por Guillén-Díaz (2010, pp. 16-17); siendo, al respecto, la recomendación de mayo de 2018 la de más reciente difusión (Comisión Europea, 2018b).

${ }^{4}$ Articulado que recogió Thünnermann (2000) en las páginas 265 y siguientes del volumen en el que realiza un balance y visión prospectiva de la Educación Superior en Latinoamérica.
} 


\title{
1. Revisión de la literatura
}

En este sentido, se demanda a esos estudiantes universitarios su implicación activa sustentada -según enuncia el Artículo 5-, por el fomento y refuerzo de la innovación, la interdisciplinariedad y transdisciplinariedad, y el incremento de la investigación en todas las disciplinas. Se incide en que:

\begin{abstract}
Reviste especial importancia el fomento de las capacidades de investigación en los establecimientos de enseñanza superior con funciones de investigación puesto que cuando la educación superior y la investigación se llevan a cabo en un alto nivel dentro de la misma institución se logra una potenciación mutua de la calidad. (p. 24)
\end{abstract}

De forma complementaria, en el otro documento citado, el Marco de Acción (UNESCO, 1998), se acuerda que los centros universitarios,

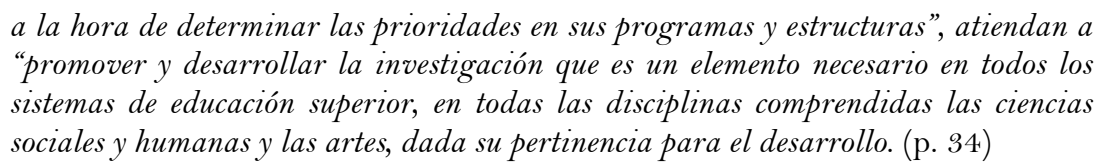

Consecuentemente, estamos asistiendo -desde la Declaración de Bolonia de 19 de junio de 19995-, a sucesivas Comunicaciones de la Comisión al Consejo y al Parlamento Europeo, orientadas a la construcción de un espacio europeo de investigación, otorgando a las Universidades el papel fundamental de velar por el funcionamiento del denominado "triángulo del conocimiento". Se instaura el debate en torno a la modernización en cuatro ámbitos científicos principales: Ciencias de la Vida/salud, Ciencias Sociales, Tecnología/informática y Ciencias Humanas/bellas artes, siendo la Educación una prioridad en esta iniciativa ${ }^{6}$.

Se trata, en el seno de ese proyecto de modernización de la Educación Superior (Comisión Europea, 2011), de reforzar las sinergias entre los polos del triángulo del conocimiento que asocia la educación/formación, la investigación y la innovación, pues “desempeña un papel fundamental para el progreso individual y societal, y para proporcionar el capital humano altamente cualificado y los ciudadanos formados que Europa necesita para generar empleo, crecimiento económico y prosperidad" (p. 2). Se requiere pues que las Universidades, en la concepción de sus Planes de estudios, tengan la capacidad de asegurar actividades de enseñanza, de investigación y el logro de los estudiantes.

En nuestro ámbito nacional, con el proceso abierto que representa el desarrollo de la Estrategia Universidad 2015 (Ministerio de Educación, 2010), se ha invitado a todos aquellos "con interés por el futuro de la Universidad como institución de educación superior, a compartir análisis, visiones, prospectivas y oportunidades además de sugerir soluciones para sus debilidades y amenazas" (p. 12). Y, se plantea que "Además, deben revalorizarse las titulaciones de Humanidades, Ciencias Sociales, Derecho y Educación con el fin de equilibrar la oferta de conocimiento necesaria para el progreso socioeconómico” (p. 26).

\footnotetext{
${ }^{5}$ Que tiene el origen, a su vez, en la Declaración de la Sorbona, adoptada en París el 25 de mayo de 1998.

${ }^{6}$ Objeto de la Conferencia The knowledge triangle shaping the future of Europe, celebraba en Göteborg del 31 de agosto a 2 de septiembre de 2009. El Informe final está alojado en la web de la Agencia Nacional Sueca para la Educación Superior.
} 


\subsection{Contextualización disciplinar de los Trabajos Fin de Grado}

Asumiendo como declaran los expertos que, en los aproximadamente 4.000 centros de Educación Superior que existen en Europa, el Grado en Maestro(a) de Primaria (Menciones de Lengua Extranjera, Inglés-Francés), tiene menos atractivo que otros, estimamos oportuno destacar que no por ello deja de ser un elemento estratégico de formación, para las funciones y tareas que se atribuyen a los egresados en materia de dominio de lenguas. Además, este Grado como otros participa de la sensibilización a la importancia de la investigación, a través del estatus otorgado a los Trabajos Fin de Grado -en adelante TFG(s)-, en la organización disciplinar por materias del Plan de estudios.

Conforme a la normativa reguladora, constituyen una asignatura del último año. Corresponden a la materia Practicum-Trabajo Fin de Grado, adscrita y asignada al área de conocimiento de Didáctica de la Lengua y la Literatura. Como aparece en la Guía docente (Universidad de Valladolid, 2018), un TFG se concibe como un trabajo escrito, "que supone la realización por parte del estudiante de un proyecto, memoria o estudio" (p. 2), siempre original e inédito en torno a un tema específico, que permita demostrar la adquisición de las competencias establecidas para un desempeño docente específico, asociado a la Mención. En este sentido, se precisa que:

La importancia de un trabajo de estas características radica en que fomenta en el
estudiante habilidades tan relevantes como ser capaz de seleccionar un tema;
planificar un proceso de análisis y estudio del tema seleccionado, estableciendo unos
objetivos para el mismo; y ofrecer y defender una respuesta lógica y justificada a los
problemas o situaciones planteados. Por tanto, la realización de este tipo de trabajos
es un mecanismo que permite desarrollar en el estudiante las capacidades de análisis,
de resolución de problemas y de asimilación y presentación de resultados, además de
las competencias indicadas expresamente en el respectivo Plan de estudios. (p. 2)

Los TFGs irrumpen en nuestra Educación Superior como documento académico de gran repercusión por su papel en la evaluación final de los estudiantes. Desde los inicios de su implantación, surgen estudios para distintas titulaciones de Ciencias Sociales y Jurídicas, como es el caso del volumen de Lazo (2010). En su mayor parte, son expuestos en reuniones científicas revelando la preocupación por las responsabilidades y perspectivas docentes abiertas en Donoso Anes y Serrano Domínguez (2014); o por su gestión institucional y organizativa en Civila (2014), y por su diseño y orientación en Fernández de Larrea y otros (2014). En estos casos, las autoras nos acercan en concreto a los Grados de Maestro(a) en sus instituciones universitarias.

En ámbitos internacionales, y siempre considerando el contexto universitario marcado por la preeminencia de la investigación en Educación, las aportaciones de Roy (1989), Shavelson y Towne (2002) y Whitty (2006), entre otros, revelan la amplitud del debate abierto respecto a prioridades de la investigación en programas y estructuras. Así lo encontramos en trabajos transferibles al caso que nos ocupa; a saber: el de Boucher (1994) centrado en la formación y acompañamiento de docentes en su desarrollo profesional; el de Coen y otros (2010) indagando en las percepciones de docentes en formación inicial; el de Hofstetter (2005) que vincula la orientación a la investigación a la profesionalización de los docentes, y el de Rey (2014) cuya expertise en la mediación entre investigación y práctica, le hace preocuparse especialmente por la cientificidad, la mejora y la incidencia de las investigaciones en Educación.

Se comparte la convicción de que no son solo los conocimientos teóricos o la experiencia práctica los componentes de base de actuaciones docentes competentes, sino más bien los 
sustentados por la investigación, por pruebas lo más científicas posible. Y, se ha cristalizado el concepto evidence based education, utilizándolo con el carácter más amplio de evidence based research, cuyas consecuencias expone Rey (2006, 2014) precisándolo como educación basada en los resultados de la investigación. Ante las dificultades de relación entre investigación y práctica educativa que ha abordado Murillo (2011), se debe evitar en los docentes el simple "rol consumidor" (p. 8), orientándoles a asumir el de generadores de conocimiento, porque "tengan formación sobre investigación educativa” (p. 10).

\subsection{Premisas}

En este sentido, en el contexto disciplinar de las Menciones, por las que se añade una especialización a estos estudiantes universitarios, estimamos oportuno sustentar que un TFG orientado a la investigación:

- Constituye un instrumento estratégico de producción de conocimiento para su comprensión y apropiación, aplicando a escala individual las habilidades competenciales relativas a métodos y procedimientos de investigación (Ministerio de Educación y Ciencia, 2007).

- Se integra en la dimensión profesional de la formación que se ofrece en el área de conocimiento de la Didáctica de la Lengua y la Literatura, como un sistema fiable, como una fase inicial significativa y clave hacia la excelencia de la carrera profesional y, eventualmente, para el paso a Planes de estudios de Master y Doctorado, promocionados para la investigación.

Esta consideración del TFG se encuentra reforzada por el contenido de documentos como el de la OCDE (2005) sobre la demanda de profesores de calidad, y el de la ya citada Conferencia de Göteborg, de 2009. De forma que se espera en formación inicial universitaria, una aportación individual mediante esa actividad científica que supone un TFG orientado a la investigación:

- Inscrito en la problemática que encierra la enseñanza-aprendizaje de las Lenguas Extranjeras (Francés-Inglés): sus contextos, métodos, procesos y materiales (línea temática propuesta en el Plan de estudios).

- Focalizado en un problema, planteando interrogantes que conduzcan a delimitar y definir un objeto de estudio propio, tal y como sugieren para la investigación en Didáctica de las Lenguas-Culturas (Blanchet y Chardonnet, 2011), y el metodólogo por excelencia de este ámbito (Puren, 2001, 2015).

- Vinculado a las condiciones del estudiante de beneficiario y agente de la importancia de la investigación en la atención a necesidades emergentes, en la mejora de su formación, y en el enriquecimiento de la profesión en sí misma, como se plantea internacionalmente en la reciente publicación de Brandenburg y otros (2016).

Como beneficiario debe adscribirse a aquellos principios que revelan esa importancia (objetiva y para sí mismo); a saber:

- La construcción de la autonomía ante sus responsabilidades, en la percepción y resolución de problemas, en la observación y comprensión de la realidad educativa y de sí mismo como profesional docente. 
- La toma de conciencia del alcance del TFG, y la visión crítica exigente ante un conocimiento producido científicamente, aplicable y adaptable a contextos específicos, situándose en la complejidad y la inter y transdisciplinariedad, argumentadas por Morin (1994) para la producción del conocimiento en Educación.

- La apropiación de bases teóricas sólidas, como recurso para regular un proceso científico de investigación integrado en el TFG, para articular teoría y práctica y, posteriormente, práctica y la reflexión sobre la práctica instaurada por Donald Schön.

Como agente, conforme a aportaciones metodológicas tales como las de Cifuentes (2013), Martínez González (2007), o Prégent (2001), entre otros, debe asegurar la orientación a la investigación a través de las tres determinaciones que subyacen a la concepción y elaboración de un TFG:

- La identificativa/decisional que correspondería al interrogante ¿qué tipo de TFG es?

- La estructural y organizativa que correspondería al interrogante ¿de qué forma se presenta y se realiza?

- La vinculatoria/valorativa que correspondería al interrogante ¿qué sentido y alcance se le otorga como beneficiario y agente?

\subsection{Componentes consustanciales del Trabajo Fin de Grado}

En torno a esas determinaciones, se articulan los componentes de un diseño de investigación observables en términos de elementos configuradores esenciales. Su presencia en el TFG permite identificar la orientación a la investigación, por cuanto que:

- La identificativa/decisional corresponde a un posicionamiento intelectual, por la toma de conciencia de que se realiza una investigación. Estará en elementos como el título, la presentación, el resumen y las palabras clave.

- La estructural y organizativa está marcada por un posicionamiento metodológico para la actividad propiamente científica. Estará en elementos como la introducción, los epígrafes dedicados a fundamentos teóricos, interrogantes, objeto de estudio y objetivos, así como en los relativos a aspectos metodológicos (contexto, muestra, tratamiento de datos, etc.), y conclusiones.

- La vinculatoria/valorativa está marcada por el posicionamiento profesional docente. Estará en epígrafes dedicados a competencias (del Título-Grado, del TFG orientado a la investigación), referencias, máximas (en el sentido de principios de acción) balance final sobre el TFG en sí mismo y su realización como futuro profesional docente.

\section{Método}

En la perspectiva tanto de la valorización de estos trabajos y su vinculación al funcionamiento del triángulo del conocimiento, como de la reputación del Grado respecto de la dimensión profesional de la formación docente, nos planteamos: i) ¿iSon los TFGs ricos en expectativas de contribución a la investigación? ii) ¿'Son valorables los TFGs como 
propios de una investigación, y a qué obstáculos eventuales se confrontan los estudiantes del Grado para realizarlos orientados a la investigación?

\section{Objetivos}

Desde las responsabilidades que conlleva la tutela de TFGs, sin entrar en cuestiones de gestión institucional, evaluación, modalidades de calificación, etc., formulamos como objetivo general: dar cuenta de la presencia y calidad de los componentes consustanciales de una investigación, en los TFGs del Grado en Maestro(a) de Educación Primaria, Menciones de Lengua Extranjera (Francés-Inglés).

Objetivo que articulamos en los objetivos específicos:

- Analizar e interpretar las informaciones textuales que aportan los elementos configuradores de esos TFGs, en relación con la orientación a la investigación.

- Caracterizar esos TFGs, por la calidad de orientación a la investigación de sus elementos configuradores, en términos de puntos fuertes y obstáculos.

\section{Muestra documental}

Para disponer de informaciones explícitas e incluso implícitas sobre elementos propios de toda investigación en los TFGs, accedemos a los de las Menciones que nos ocupan. Depositados en el Departamento de Didáctica de la Lengua y la Literatura de la Facultad de Educación y Trabajo Social, desde el curso 2010-2011 en soporte papel y digital, son documentos de acceso inmediato y fácil manejo. Nos ofrecen un conjunto de informaciones textuales escritas emitidas individualmente, con carácter de datos invocados cuya constitución es anterior (incluso exterior) a nuestro estudio (Van der Maren, 1996).

Siguiendo el criterio dominante de la disponibilidad al que alude Cea (2001), y de una muestra no probabilística conveniente para profundizar en las informaciones, se seleccionan TFGs de egresados que finalizaron sus estudios dentro los cuatro años del Plan de estudios y, por tanto, realizados simultáneamente a la otra asignatura de la materia: el Practicum.

Cuadro 1. La muestra de TFGs, por Curso académico y Menciones de Lengua Extranjera

\begin{tabular}{cccc}
\hline $\begin{array}{c}\text { CURSO } \\
\text { ACADÉMICO }\end{array}$ & $\begin{array}{c}\text { Total 180 } \\
\text { TFGs }\end{array}$ & $\begin{array}{c}\text { TFGS MENCIÓN LENGUA } \\
\text { EXTRANJERA INGLÉS }\end{array}$ & $\begin{array}{c}\text { TFGS MENCIÓN LENGUA } \\
\text { EXTRANJERA FrANCÉS }\end{array}$ \\
\hline $2011-12$ & 16 & 15 & 1 \\
$2012-13$ & 33 & 32 & 1 \\
$2013-14$ & 43 & 40 & 3 \\
$2014-15$ & 44 & 43 & 1 \\
$2015-16$ & 40 & 39 & 1 \\
$2016-17$ & 4 & 3 & 1 \\
\hline
\end{tabular}

Fuente: Elaboración propia.

La muestra es de 180 documentos. Su descripción en el cuadro 1, por curso académico y Lengua Extranjera de la Mención, revela la fuerte demanda de la de inglés derivada de la tendencia social a valorar el dominio del inglés sobre el francés, a diferencia de décadas anteriores. 


\section{Instrumento}

Establecemos un sistema categorial que, en torno a las determinaciones descritas, reúne los elementos configuradores de una investigación definidos en 1.2. Premisas. Expuesto en el cuadro 2 que sigue, es la base para elaborar las parrillas utilizables en el análisis de los TFGs, a la búsqueda de índices cualitativos e incluso cuantificables (unidades de registro). Su validez procede del marco teórico de la investigación, y del juicio de expertos. Aportaron la sugerencia de incluir también la categoría Conclusiones en la Determinación vinculatoria/valorativa, pues aporta información adicional sobre el alcance del TFG y sus efectos en la condición del estudiante como futuro profesional de la docencia.

Cuadro 2. Determinaciones y elementos para categorías de análisis de TFGs orientados a la investigación

\begin{tabular}{|c|c|c|}
\hline $\begin{array}{c}\text { DET. IDENTIFICATIVA / } \\
\text { DECISIONAL }\end{array}$ & $\begin{array}{l}\text { DET. ESTRUCTURAL Y } \\
\text { ORGANIZATIVA }\end{array}$ & $\begin{array}{c}\text { DET. VINCULATORIA / } \\
\text { VALORATIVA }\end{array}$ \\
\hline Título & Introducción/Fundamentación & Competencias \\
\hline Presentación & Objeto de estudio/tema & Referencias \\
\hline Resumen & Objetivo General (G) & Máximas \\
\hline \multirow[t]{4}{*}{ Palabras clave } & Objetivos Específicos (Es) & Conclusiones \\
\hline & Aspectos metodológicos & Otros \\
\hline & Conclusiones & \\
\hline & Otros & \\
\hline
\end{tabular}

Fuente: Elaboración propia.

Procedimiento de recogida y análisis de datos

Desde un enfoque descriptivo e interpretativo, y adoptando un posicionamiento prospectivo y moderadamente crítico, optamos para el análisis documental por un estudio cualitativo y por la técnica global del análisis de contenido. Su pertinencia y adecuación, avaladas por Bardin (2002), Krippenforff (1990) y Miles y Huberman (1994) al tratarse de informaciones textuales escritas, residen en:

- La necesaria sistematicidad, pues las informaciones están muy marcadas por la demanda de individualidad en la realización del TFG; por condicionantes de autoría única, tema original respecto de la línea temática enunciada en 1.1. Contextualización disciplinar de los Trabajos Fin de Grado; por la referencia a situaciones particulares según tipo de alumnos, centros y niveles educativos, etc.

- La aproximación al corpus de datos, según dos niveles analíticos de lectura y varias lecturas, obteniendo el contenido explícito y, si fuese el caso, el implícito, mediante el tratamiento semántico manual que favorece la reflexión e implicación de los investigadores, en un estudio más valorativo que evaluativo (siguiendo el posicionamiento experimentado de Wanlin, 2007).

- La recogida de datos hasta el punto de saturación, pues, en el manejo de la muestra, para los elementos retenidos como unidades de análisis, no se ofrecían unidades de registro nuevas, ni se observaban otros. 
Cuadro 3. Datos ilustrativos del análisis de la determinación identificativa/decisional, en los TFGs del 2011 al 2014 y del 2015 al 2017

\begin{tabular}{|c|c|}
\hline $\begin{array}{c}\text { UNIDADES DE } \\
\text { ANÁLISIS }\end{array}$ & UNIDADES DE REGISTRO \\
\hline \multicolumn{2}{|c|}{ TFGs del 2011 al 2014} \\
\hline Título & 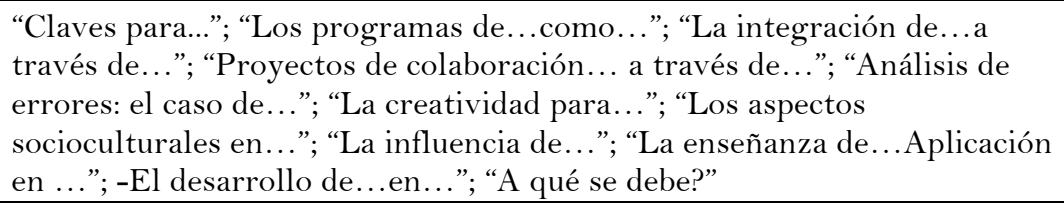 \\
\hline Presentación & $\begin{array}{l}\text { "Con el título...y dirigido por..."; "Al término de mis estudios...recoge las } \\
\text { competencias" }\end{array}$ \\
\hline Resumen & 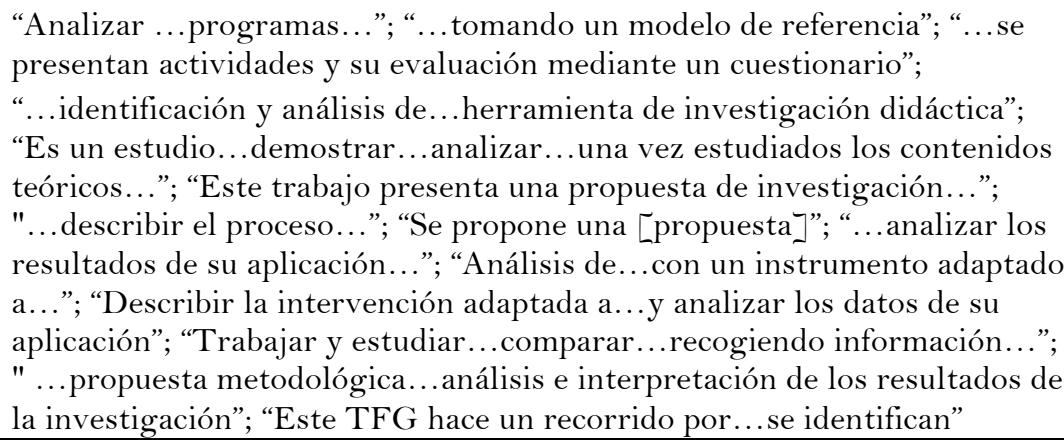 \\
\hline Palabras clave & $\begin{array}{l}\text { Análisis de errores; Clasificación; Parrilla de evaluación; Análisis de } \\
\text { contenido; } \\
\text { Análisis de objetivos }\end{array}$ \\
\hline \multicolumn{2}{|c|}{ TFGs del 2015 al 2017} \\
\hline Título & 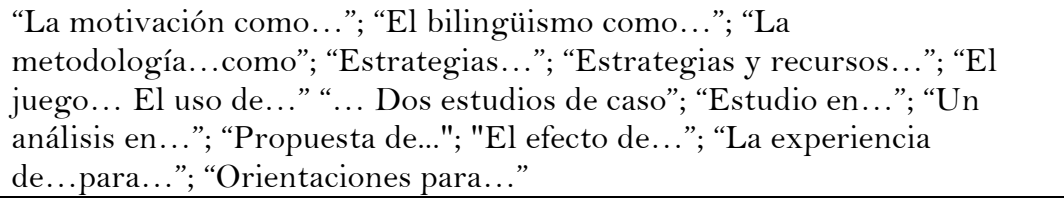 \\
\hline Presentación & "...poner de manifiesto los conocimientos que he adquirido" \\
\hline Resumen & 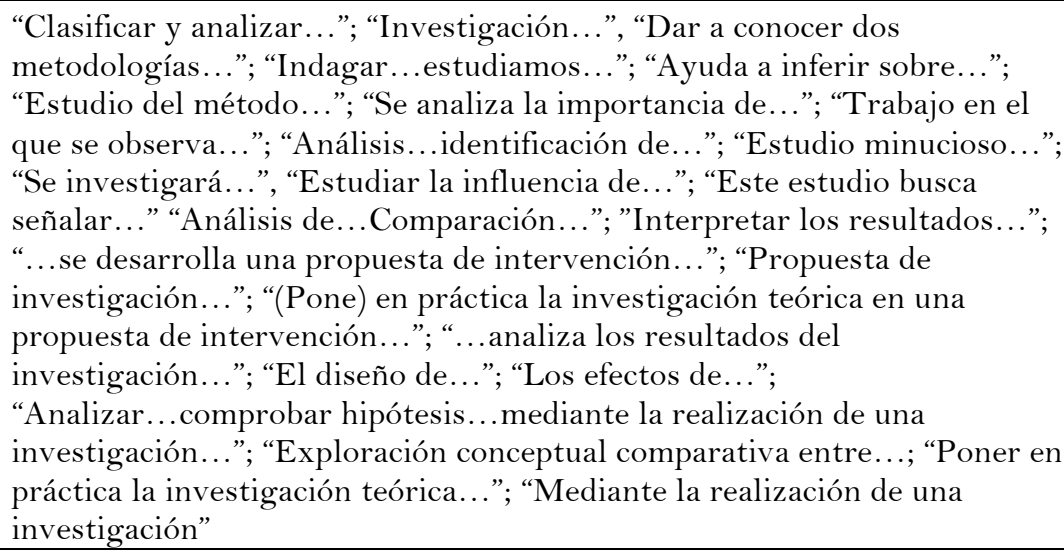 \\
\hline Palabras clave & Observación; Cuestionario; Motivación; Actuación docente \\
\hline
\end{tabular}

Fuente: Elaboración propia.

Para el análisis de los datos y la interpretación de los resultados se aplican los procedimientos de anonimización, conforme al rigor buscado en este estudio. Y, para una visión longitudinal en la búsqueda de índices significativos, quizá evolutivos, los TFGs se agrupan en dos bloques cronológicos: 92 TFGs, del 2011 al 2014, y 88 TFGs, del 2015 al 2017. 
Cuadro 4. Datos ilustrativos del análisis de la determinación estructural y organizativa, en los TFGs del 2011 al 2014 y del 2015 al 2017

\begin{tabular}{|c|c|}
\hline $\begin{array}{l}\text { UNIDADES DE } \\
\text { ANÁLISIS }\end{array}$ & UNIDADES DE REGISTRO \\
\hline \multicolumn{2}{|c|}{ TFGs del 2011 al 2014} \\
\hline $\begin{array}{l}\text { Introducción/ } \\
\text { Fundamentación }\end{array}$ & $\begin{array}{l}\text { “...se tratan las características y la implantación de...”; “Se pretende } \\
\text { abordar...ventajas e inconvenientes en...”; "Se trata de un trabajo de } \\
\text { investigación...”; "Se realizará un estudio sobre la ...”; "Observo, recojo, } \\
\text { analizo" }\end{array}$ \\
\hline $\begin{array}{l}\text { Objeto de } \\
\text { estudio/tema }\end{array}$ & $\begin{array}{l}\text { "Buscar soluciones...”; "los errores...”; "El ámbito del bilingüismo...”; } \\
\text { "Las realidades de...”; "Las estrategias didácticas...”; "Indagar en...”; ... }\end{array}$ \\
\hline \multirow{3}{*}{$\begin{array}{l}\text { Objetivo } \\
\text { General }(\mathrm{G}) \text { y } \\
\text { Específico }(\mathrm{Es})\end{array}$} & $\begin{array}{l}(G) \text { "Proponer y defender los programas de inmersión...”; Conocer } \\
\text { expectativas y...”; "Desarrollar en los alumnos...”; "Presentación de } \\
\text { actividades propuestas...”; “Categorizar los errores...”; "Realizar un } \\
\text { acercamiento al estudio de los beneficios de...”; “Diseñar, planificar, } \\
\text { adaptar y evaluar una propuesta de intervención”; “Fomentar la } \\
\text { creatividad de los alumnos" }\end{array}$ \\
\hline & $\begin{array}{l}\text { (Es) “Demostrar factores clave...”; “Extraer conclusiones que...”; } \\
\text { "Analizar...comprobar...”; “Analizar...Valorar...”; “Analizar y evaluar...”; }\end{array}$ \\
\hline & $\begin{array}{l}\text { "Analizar ... Extraer conclusiones...”; "Poner en práctica el desarrollo } \\
\text { de...”; "Analizar...identificar...”; "Analizar mi propia experiencia” }\end{array}$ \\
\hline $\begin{array}{l}\text { Aspectos } \\
\text { metodológicos }\end{array}$ & $\begin{array}{l}\text { "Contexto; "Observación y cuestionario"; Entrevistas y cuestionario a } \\
\text { agentes educativos; "Parrillas de análisis; "Muestra"; "Cuestionario y } \\
\text { gráfica comparativa"; "Soportes, ordenadores"; "Resultados en gráficas"; } \\
\text { "Gráficos de frecuencias"; "Fases de la investigación”; "Investigación } \\
\text { cuantitativa”; "Descripción del proceso"; "Comparativa"; "Limitaciones del } \\
\text { instrumento"; "Tablas comparativas"; "Estudio de casos; "Cualitativo" }\end{array}$ \\
\hline Conclusiones & $\begin{array}{l}\text { "Nos aporta una visión más amplia...”; “Toma de conciencia de la } \\
\text { dificultad de la práctica de aula...”; "Ha supuesto un paso más en la } \\
\text { formación a nivel profesional..."; "El factor creativo del trabajo” }\end{array}$ \\
\hline \multicolumn{2}{|c|}{ TFGs del 2015 al 2017} \\
\hline $\begin{array}{l}\text { Introducción/ } \\
\text { Fundamentación }\end{array}$ & 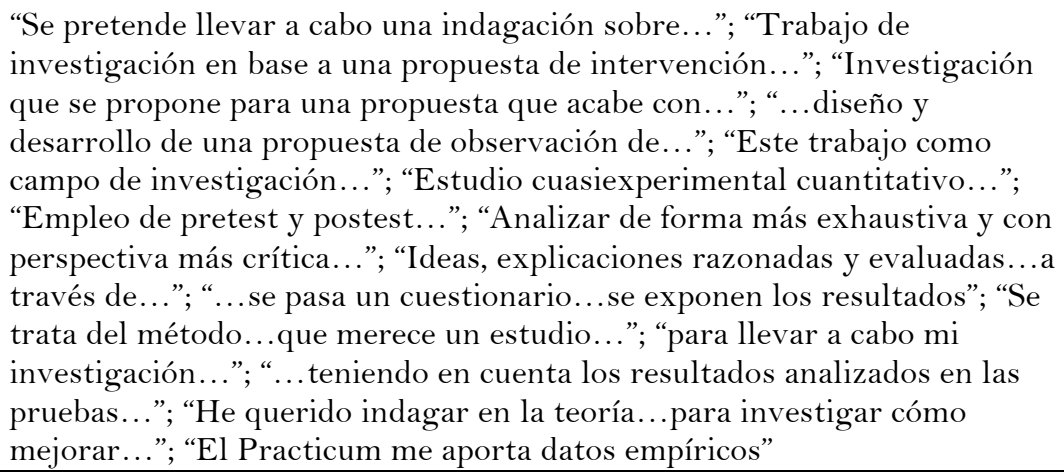 \\
\hline $\begin{array}{l}\text { Objeto de } \\
\text { estudio/tema }\end{array}$ & $\begin{array}{l}\text { "Ventajas y desventajas de algunos aspectos del proceso"; “motivación”; "el } \\
\text { éxito escolar"; "estrategias didácticas"; “Análisis de profesorado"; "mejoras } \\
\text { de carácter práctico"; "Arrojar algo de luz"; Analizar la situación actual”; } \\
\text { "Determinar ciertos aspectos"; "Identificar puntos fuertes y débiles" }\end{array}$ \\
\hline $\begin{array}{l}\text { Objetivo General } \\
(\mathrm{G}) \\
\text { Específico }(\mathrm{Es})\end{array}$ & $\begin{array}{l}\text { (G) "Ofrecer un panorama general de las enseñanza identificando } \\
\text { similitudes y diferencias"; "Dar cuenta de las estrategias de... en la } \\
\text { intervención del docente y observar las de aprendizaje..."; "Fundamentar } \\
\text { la importancia que tienen las estrategias..."; "Dar cuenta de los beneficios } \\
\text { e inconvenientes de la enseñanza de la LE" "Mejorar la formación del } \\
\text { profesorado"; "Conocer la realidad del uso de la motivación"; "Determinar } \\
\text { relación entre motivación y estrategias didácticas"; "Indagar en los efectos } \\
\text { de los programas de inmersión en el proceso de construcción de } \\
\text { conocimiento de la DNL" }\end{array}$ \\
\hline
\end{tabular}


(Es) "Observar actividades y ejercicios sobre las ciencias naturales y artes plásticas para comprobar la movilización de unas estrategias específicas”; "Descubrir la capacidad e interés de motivación del profesorado...introducción de nuevos recursos motivadores"; "Valorar y conocer los componentes observables de la competencia emocional del alumnado"; "Valorar vinculación entre TIC y mayor motivación"; "Comprobar si influye en el éxito escolar...”; "Profundizar en...”; “Analizar el juego...”; "Diseñar tipos de juego...”; "Observar la respuesta de los alumnos"

“Contextualización”; "Rigurosa descripción contextual; "Hipótesis y variables; "Muestra”; "Corpus documental; "Recogida de datos”; "Observación y recogida de información”; "Cuestionario”; "Grabaciones”; Aspectos "Hoja de registro; "Notas de campo"; "Encuesta electrónica”; "Gráficos y metodológicos tablas de resultados"; "Diagrama porcentual; "Resultados descriptivos, cuantitativos..." "Tratamiento cuantitativo"; "Estudio cualitativo"; "Diseño etnográfico”; “Temporalización...”; "Método comparativo”; Escala Likert”; Observación directa”; "Porcentajes"; "Pretest y postest validados"; "Análisis de resultados medias y varianzas"; "Sacando evidencias"

"La realización supone el broche final de la Mención...”; "Una

Conclusiones $\quad$ oportunidad de conocer..."; "Estoy satisfecha del trabajo..."; "Futuras líneas..."; "Se han desarrollado las competencias específicas del Grado"

Fuente: Elaboración propia.

Cuadro 5. Datos ilustrativos del análisis de la determinación vinculatoria/valorativa, en los TFGs del 2011 al $2014 \mathrm{y}$ del 2015 al 2017

\begin{tabular}{|c|c|}
\hline $\begin{array}{c}\text { UNIDADES DE } \\
\text { ANÁLISIS }\end{array}$ & UNIDADES DE REGISTRO \\
\hline \multicolumn{2}{|c|}{ TFGs del 2011 al 2014} \\
\hline Competencias & $\begin{array}{l}\text { "Comunicar conocimientos y compromiso ético”; “Demostrar los } \\
\text { conocimientos y capacidades adquiridas del Grado Mención”; "Las } \\
\text { específicas del Grado” }\end{array}$ \\
\hline Máximas & $\begin{array}{l}\text { “... ayuda al profesorado en activo...”; ...otras perspectivas observando...”; } \\
\text { "Perspectiva analítica y crítica de gran importancia” }\end{array}$ \\
\hline Conclusiones & $\begin{array}{l}\text { "He podido ver una realidad muy diferente"; "He cambiado mi concepción de } \\
\text { la educación y la implicación laboral" }\end{array}$ \\
\hline \multicolumn{2}{|c|}{ TFGs del 2015 al 2017} \\
\hline Competencias & $\begin{array}{l}\text { “Transdisciplinariedad”; Desarrollar pensamiento crítico”; “...necesidad de } \\
\text { comprender y analizar las informaciones”; Incremento del grado de } \\
\text { adquisición de las competencias del título; “....una realidad diferente”; } \\
\text { "Oportunidad de abrir la mente”; “Actualizarse y hacer síntesis de la } \\
\text { investigación”; "Implementación de un proceso de investigación es vital...”; } \\
\text { "Interpretación de datos, emitir juicios prácticos" }\end{array}$ \\
\hline Máximas & $\begin{array}{l}\text { "Actualizar su forma de motivar...”; "La labor del docente es actualizarse”; } \\
\text { "Punto de vista más objetivo”; “Oportunidad de conocer”; “...de poner en } \\
\text { práctica”; “...el interés de seguir aprendiendo y formándose para el propio } \\
\text { avance” }\end{array}$ \\
\hline Conclusiones & $\begin{array}{l}\text { "He podido corroborar la teoría con la práctica”; "La puesta en práctica de la } \\
\text { propuesta... ha sido muy enriquecedora”. "Mi objetivo ha sido investigar } \\
\text { cómo mejorar las habilidades”; “... satisfacción del trabajo... uso de } \\
\text { conocimientos previos”; “... diversidad de opiniones sobre la } \\
\text { internacionalización educativa" }\end{array}$ \\
\hline
\end{tabular}

Fuente: Elaboración propia.

Diseñamos las parrillas del análisis propiamente dicho, consignando para cada determinación la Unidades de análisis y Unidades de registro. A título ilustrativo, en los 
respectivos cuadros 3,4 y 5 aportamos algunos datos registrados mediante la aplicación del análisis de contenido.

\section{Resultados}

Profundizamos en el análisis de contenido, ofreciendo una visión cuantitativa, descriptiva e interpretativa de los resultados.

\subsection{La distribución porcentual}

La visión cuantitativa de la presencia de los elementos configuradores de la investigación ofrecida en el cuadro 6, permite comparar los dos bloques cronológicos de TFGs.

Cuadro 6. Distribución de los elementos configuradores de la investigación en los TFGs (en porcentaje)

\begin{tabular}{clrr}
\hline & & 2011-2014* & 2015-2017** \\
\hline \multirow{2}{*}{ Identificativa / } & Título & 22,8 & 36,4 \\
Decisional & Resentación & - & - \\
& Palabras clave & 39,1 & 42 \\
& Introducción & 3,7 & 4,5 \\
\hline \multirow{2}{*}{ Estructural y } & Objeto de estudio & 5,4 & 33 \\
organizativa & Objetivos & 12 & 35,2 \\
& Aspectos metodológicos & 68,5 & 81,8 \\
& Conclusiones & 25 & 43,2 \\
\hline \multirow{2}{*}{ Vinculatoria / } & Competencias & - & - \\
Valorativa & Referencias & 3,3 & 8 \\
& Máximas & - & - \\
& Conclusiones & 5,4 & 20,5 \\
\hline
\end{tabular}

Nota: - No contabilizable. ${ }^{*}$ El total de TFGs del periodo $2011-2014$ es 92. ** El total de TFGs del periodo 2015-2017 es 88 .

Fuente: Elaboración propia.

El aumento relativo de la orientación a la investigación en los TFGs del 2015 al 2017 queda patente con las diferencias porcentuales de presencia de los elementos siguientes: Introducción y Objeto de estudio, y Máximas y Conclusiones. Traducen una evolución, respectivamente, en las determinaciones estructural y organizativa y vinculatoria/ valorativa. Podemos inferir que es debida, a priori, a un aumento en ese período de las habilidades competenciales de orden estructural y organizativo, aun cuando en ambos bloques los resultados de la dimensión identificativa/decisional sean más semejantes.

\subsection{La determinación identificativa/decisional}

Si bien, el elemento Presentación es de calidad nula para dar cuenta de la orientación a la investigación de los TFGs, al dedicarse a contextualizar el documento indicando el Grado, Tutor, naturaleza de la asignatura y vinculación a la línea de trabajo, podemos interpretar que:

- El Título es elemento de calidad media-alta en los dos bloques de TFGs, por presentarse de forma implícita, ya sea utilizando un interrogante, ya sea con términos como el uso de, hacia, etc., siempre marcando la utilidad con: para, como, a través de, en. Y, porque revela solamente una atención a temas o centros 
de interés: los aspectos socioculturales; la metodología CLIL, las estrategias y recursos, las actividades comunicativas, los materiales, etc.

- El Resumen es elemento de calidad alta en los dos bloques, al presentar procedimientos, operaciones e instrumentos propios de un proceso de investigación, aun cuando no en todos de una forma completa y explícita que daría cuenta del rigor científico esperado. Desde los términos: investigación didáctica, estudio, propuesta de investigación, el diseño de, etc., se concreta: trabajar y estudiar, interpretación de los resultados de la investigación, identificación, demostrar, describir (la intervención), analizar los datos de su aplicación. En los del 2015 al 2017 se alude además a: estudio minucioso, clasificar y analizar, indagar, comprobar hipótesis, identificación, se observa cómo, comparación. Y, aun con vinculación a la práctica, a la intervención didáctica mediante precisiones de qué se trata: de propuestas metodológicas, de intervención, así como de actividades, y de aplicaciones, que para algunos conllevan una evaluación, pero realizada por parte de los alumnos destinatarios.

- Las Palabras clave son, en los dos bloques, elementos de calidad baja; pero, siendo muy pocas, sí indican la orientación a la investigación por acciones o por procedimientos e instrumentos.

\subsection{La determinación estructural y organizativa}

La Introducción es elemento de calidad baja en el bloque del 2011 al 2014, evidenciada ya con el bajo porcentaje de presencia $(5,4 \%)$, por ausencia total de interrogantes, aun ofreciendo una breve justificación de las competencias a las que se atiende en su realización. Se alude, por ejemplo, a: Los conocimientos adquiridos durante cuatro años...han hecho posible elaborar el TFG; a que Se aplican los conocimientos adquiridos, mostrando las competencias del Grado. En el bloque del 2015 al 2017, este elemento es de calidad media-alta, por términos que, en todo caso, son recurrentes: investigación, mi investigación, estudio, estudio de caso; pero que anticipan una orientación a la investigación: observación, proceso de indagación a través, de empleo de, he analizado, se exponen los resultados, análisis para las conclusiones, etc.

El Objeto de estudio es elemento de calidad baja en ambos bloques. En el del 2011 al 2014, por imprecisiones al indicar: soluciones, errores, el ámbito del bilingüismo, las realidades, etc. Y, en el bloque del 2015 al 2017 , porque se identifica difícilmente ante términos como: ventajas y limitaciones, dificultades, la efectividad de, potencialidades de, etc., y porque reproduce objetivos de la Guía docente del TFG (como asignatura), incluyendo procedimientos de investigación.

Los Objetivos, tanto el general como los específicos, en el bloque del 2011 al 2014, son de calidad media, apoyada por el alto porcentaje de presencia $(68,5 \%)$, y porque aun habiendo algunos orientados a la investigación, otros son formulados desde lo que se concibe como propuesta y diseño de actividades para los escolares: Desarrollar en los alumnos...; e incluso, desde la propia intervención docente: Ser capaz de promover... en los alumnos, o de analizar mi propia experiencia. En el bloque del 2015 al 2017, estos elementos son de calidad muy alta porque permiten interpretar una toma de conciencia del alcance académico-disciplinar del TFG orientado a la investigación: fundamentar, ofrecer un panorama general de, profundizar, delimitar un marco referencial, analizar estudios sobre, definir, describir y estudiar el concepto de, etc. Al mismo tiempo, porque describen el carácter de utilidad que se esperaría de toda investigación: conocer la realidad, contribuir 
a la optimización de, fomentar el uso de, observar para, descubrir para introducir nuevos recursos, mejorar la formación del profesorado, crear una base que sirva a (los maestros), etc.

Los Aspectos metodológicos, en el bloque del 2011 al 2014, son de calidad media-baja, porque están escasamente pormenorizados, limitados al contexto y a instrumentos: cuestionarios, entrevistas a alumnos, parrillas de análisis; y porque retoman los objetivos, o anticipan unas conclusiones aludiendo a limitaciones de tiempo, situación, etc. En el bloque del 2015 al 2017, estos elementos son de calidad alta porque se identifican significativos indicadores de acciones científicas de investigación: observar, analizar, describir, clasificar, recoger, evaluar, calibrar el instrumento de análisis, etc. En todos los TFGs de este bloque se describe el contexto (de la experiencia, la práctica, etc.) y se alude a metodología del trabajo, e incluso a un balance crítico del proceso de investigación. De forma particular, encontramos: muestra, (piloto-control), cuestionarios, tablas, gráficas de resultados, cuantitativos, cualitativos, descriptivos, etc., aun cuando por su carácter confuso puedan escapar en su formalización al rigor científico deseado.

Las Conclusiones, desde esta determinación, en ambos bloques son de calidad nula para dar cuenta de la orientación a la investigación de estos TFGs. Se dedican a exponer un itinerario standard para su desarrollo; por tanto, hay ausencia de los índices esperados.

\subsection{La determinación vinculatoria/valorativa}

Las Competencias, en ambos bloques, se considerarían elemento de calidad baja porque reproducen las de la Guía docente del TFG (como asignatura). La valoración de una movilización de habilidades propias de una investigación es, en cualquier caso, muy reducida; en concreto, en el bloque 2015-2017, se alude sucintamente a la capacidad de análisis e interpretación de resultados.

Las Referencias, en ambos bloques, son de calidad nula para dar cuenta de la orientación a la investigación de estos TFGs, por ausencia de vinculación explícita a la investigación. Con carácter implícito, observamos una sola referencia en 2 casos del bloque 2015 al 2017. Cabría pensar que es un elemento intencionalmente poco atendido o extenso, por el requisito del número de páginas que podría resultar escaso para cada documento.

Las Máximas, en el bloque del 2011 al 2014, son de calidad muy baja, ya evidenciada desde el escaso porcentaje de presencia $(5,4 \%)$, y sin explicitación. Es singular el enunciado: dando un paso más en este trabajo se podría... En el bloque del 2015 al 2017, son de calidad media-alta, porque se alude explícitamente a la indagación conceptual, al conocimiento de teorías y de la realidad del aula, a visión más realista, internacional, a haber afianzado conocimientos teóricos, cambio de preconcepciones, etc. Y, de forma implícita, a más líneas de trabajo que me ayuden a conocer mejor la realidad educativa para. Se confirma esa calidad por enunciados relativos a: una evolución en la propia realización del TFG, la oportunidad de conocer y comunicar con otros docentes, la gran importancia de la formación del docente en un espectro internacional.

Las Conclusiones, desde esta determinación, en ambos bloques se considerarían de calidad media, porque esbozan efectos en la condición de futuro docente: el TFG me ha permitido, me ha ayudado; supone; y aludiendo a una visión más internacional, una alternativa, beneficios de; base necesaria, etc. 


\section{Discusión y conclusiones}

Si bien, el estudio revela las limitaciones de no disponer de los beneficiarios y agentes por ser sujetos egresados, ni de Tutores por la eventualidad de su situación contractual, y de haber dejado pendiente la triangulación de las informaciones administrando encuestas y entrevistas de explicitación, exponemos algunos aspectos conclusivos. Los destacamos en términos de puntos fuertes, obstáculos y perspectivas en relación, por una parte, con la valorización y vinculación del TFG al funcionamiento del triángulo del conocimiento, y por otra, con la reputación del Grado en la óptica de la formación profesional docente.

Puntos fuertes

- La trayectoria de búsqueda de cientificidad de los TFGs, siempre en evolución, con fuerte tendencia a la mejora de la calidad de sus componentes consustanciales.

- El esfuerzo invertido especialmente en la dimensión estructural y organizativa, por la dinámica de profundización teórico-conceptual instaurada a lo largo del tiempo, y por la completitud y calidad de los componentes configuradores de la investigación.

- Las concepciones que subyacen a las dimensiones estructural y organizativa y vinculatoria/valorativa evidencian la reducción de esa separación entre concepción teórico-científica del trabajo universitario, y concepción prácticaacientífica del trabajo universitario en Educación. Se van a poder construir actividades de enseñanza a partir de TFGs de investigación.

Se abren pues expectativas para su vinculación al funcionamiento del triángulo del conocimiento, porque en la vía de la orientación a la investigación de los TFGs de este Grado, los estudiantes se inscriben en un proceso de reflexión-anticipación y acciónreflexión, situando la relación entre formación e investigación en el centro mismo de la práctica de enseñanza. Pasarían a la lógica de la utilidad del conocimiento producido, se confrontarían a la innovación conectando con la realidad educativa del destino profesional. Así se contempla ya, en la perspectiva del 2030 (OCDE 2018).

\section{Obstáculos}

Los TFGs, al ser parte de la organización disciplinar del Grado y cursarse simultáneamente el Practicum, inclinan con gran intensidad a los estudiantes hacia la primera experiencia profesional de enseñanza en los contextos escolares; cuando deberíamos aprovecharlo como un dispositivo híbrido de adquisición y producción científica de conocimientos. Se trataría de facilitar su estructura y organización para asegurar la proximidad entre el mundo académico (aspectos teórico-conceptuales) y el mundo docente (aspectos empíricos).

Estos TFGs proceden y participan, como ocurre en Educación y en el ámbito científico de la Didáctica de la Lengua y la Literatura, de esa constante o propiedad de su modo de funcionamiento que es la paradoja (Guillén-Díaz, 1999). Sucede que la investigación evoca el trabajo de equipos y en equipo, y un TFG se realiza a escala individual, cuando ahora más que nunca, se necesitaría crear las condiciones de realización en grupos de trabajo que, en este momento, la normativa considera como una excepcionalidad. Los beneficios de esa dinámica investigadora, fuertemente demandada en la formación continua docente, se extenderían a un mayor número de estudiantes, se evitarían objetivos en los mismos 
términos o muy parecidos relativos a un mismo objeto de estudio, sin tenerse en cuenta entre sí, sin dotar a los resultados de carácter "acumulativo”.

Se perciben carencias relativas a una toma de conciencia de que todo TFG podrá orientarse a la investigación, a un marco académico de calidad coherente, referido de forma más crítica a los estándares científicos de orden teórico-conceptual, base del rigor que debe caracterizar a la determinación estructural y organizativa de todo trabajo científico. Su efecto principal se dejará sentir en el reconocimiento, por parte de la comunidad científica de la Didáctica de la Lengua y la Literatura y de las instancias administrativas, de que se trata de un Grado para una formación profesionalizadora especializada (Boucher, 1994).

\section{Algunas perspectivas}

En la línea de las determinaciones de internacionalización, regionalización y mundialización, nueva dinámica de la Educación Superior y la investigación, es oportuno incidir en:

La posibilidad de ampliar el estudio a otros contextos universitarios y sujetos implicados en los TFGs, por la concertación entre diferentes instancias, con importantes efectos sobre una cultura científica común compartida (Feuer, Towne y Shavelson, 2002), y sobre la auto-regulación de la comunidad de formadores-investigadores en Didáctica de la Lengua y la Literatura, y de estos futuros docentes-investigadores, principales protagonistas de la Educación Superior.

La iniciativa de configuración de un laboratorio de acciones de investigación y conocimientos especializados en el seno los TFGs relativos a las Menciones citadas. La creación de una red de trabajos, desde el término red social cuyo papel analiza Sañudo (2012), constituye una estrategia transversal a la internacionalización in situ (en casa). Como se precisa en el Grupo de Trabajo de Internacionalización de Universidades (2014), habría de "incardinarse en la Estrategia Española de Ciencia y Tecnología y de Innovación 2013-2020 del ministerio de economía y competitividad” (p. 23). Al mismo tiempo, constituiría un factor de refuerzo del servicio de la Universidad como proveedora de proyectos.

\section{Referencias}

Bardin, L. (2002). Análisis de contenido. Madrid: Akal.

Blanchet, P. y Chardenet, P. (Dirs.). (2011). Guide pour la recherche en didactique des langues et des cultures. Approches contextualisées. París: Éditions des Archives Contemporaines.

Boucher, L. (1994). La pertinence de la recherche en éducation: état de la situation et propositions. Revue des Sciences de L'Éducation, 20(3), 563-577. https://doi.org/10.7202/031742ar

Brandenburg, R., McDonough, S., Burke, J. y White, S. (Eds.). (2016). Teacher education. innovation, intervention and impact. Singapore: Springer.

Cea, M. A. (2001). Metodología cuantitativa: Estrategias y técnicas de investigación social. Madrid: Síntesis.

Cifuentes, P. (2013). Competencias para la investigación. Papeles Salmantinos de Educación, 17, 61100.

Civila, A. (2014). Gestión de unos 800 trabajos fin de grado en una facultad de ciencias de la educación. En K. Pérez Urraza (Coord.), Actas del I congreso interuniversitario sobre el trabajo 
fin de grado. Retos y oportunidades del TFG en la sociedad del conocimiento (pp. 356-365). Bilbao: Servicio Editorial de la Universidad del País Vasco.

Coen, P. F., Pineiro, C., Herklots, L. y Haymoz, J. Y. (2010). La recherche: son image et son rôle dans la formation initiale des enseignants. En B. Wentzel y M. Mellouki (Eds.), Recherche et formation à l'enseignement: Spécificités et interdépendance (pp. 37-53). Biel: HEP-BEJUNE.

Comisión Europea. (2011). Comunicación de la comisión al parlamento europeo, al consejo, al comité económico y social europeo y al comité de las regiones. Recuperado de http://eurlex.europa.eu/legal-content/ES/TXT/PDF /? uri=CELEX:52011DC0567\&from=EN

Comisión Europea. (2018a). Recomendación del Consejo de 22 de mayo de 2018 relativa a las competencias clave para el aprendizaje permanente. Recuperado de https://eurlex.europa.eu/legal-content/ES/TXT/PDF/?uri=CELEX:32018H0604(01)\&from=LT

Comisión Europea. (2018b). Propuesta de Recomendación del Consejo relativa a un enfoque global de la enseñanza y del aprendizaje de idiomas. Recuperado de https://eurlex.europa.eu/resource.html? uri=cellar:6eb879b9-6fb4-1 1e8-9483-0 1 aa75ed 71 a 1.0022.01 $/$ DOC_1\&format $=$ PDF

Donoso Anes, J. A. y Serrano Domínguez, F. (2014). Los trabajos de fin de grado: Reflexiones y perspectivas de futuro. En E. M. Buitrago Esquinas, M. J. Sánchez Franco y R. Yñíguez Ovando (Comp.), VI jornadas de innovación e investigación docente (pp. 6-25). Sevilla: Copiarte.

Fernández de Larrea, E., Pérez Urraza, K., Bilbao Bilbao, B. y Ruiz de Gauna, P. (2014). Diseño y gestión del TFG en la escuela de magisterio de Bilbao. En K. Pérez Urraza, B. Bilbao Bilbao, E. Fernández de Larrea, B. Molero Otero y P. Ruiz de Gauna (Coords.), Actas del I congreso interuniversitario sobre el trabajo fin de grado. Retos y oportunidades del TFG en la sociedad del conocimiento (pp. 77-85). Bilbao: Servicio Editorial de la Universidad del País Vasco.

Feuer, M. J., Towne, L. y Shavelson, R. J. (2002). Scientific culture and educational research. Educational Researcher, 31(8), 4-14. https://doi.org/10.3102\%2 F0013189X031008004

Galisson, R. (1990). Où va la didactique du français langue étrangère? Études de Linguistique Appliquée. Revue de Didactologie des langues-cultures, 79, 9-28.

Grupo de Trabajo de Internacionalización de Universidades. (2014). Estrategia para la internacionalización de las universidades españolas 2015-2020. Madrid: Ministerio de Educación, Cultura y Deporte.

Guillén-Díaz, C. (1999). El área de didáctica de la lengua y la literatura. Identidad y entidad de una disciplina específica. Lenguaje y Textos, 13, 11-27.

Guillén-Díaz, C. (2010). L'éducation et la formation langagière dans la perspective curriculaire européenne. En C. Guillén (Coord.), Francés. Complementos de formación disciplinar (pp. 1338). Barcelona: Graó.

Hofstetter, R. (2005). La professionnalisation des enseignants à travers une initiation à la recherche. Le mémoire comme formation à et par la recherche de la Section des sciences de l'éducation de l'Université de Genève. Formation et Pratiques D'Enseignement en Questions, 2, 71-89.

Krippendorff, P. (1990). Metodología del análisis de contenido. Teoría y práctica. Barcelona: Paidós.

Lazo, M. C. (Coord.). (2010). El EEES y el proyecto final en los grados de comunicación. Madrid: Fragua.

Martínez González, R. A. (2007). La investigación en la práctica educativa: Guía metodológica de investigación para el diagnóstico y evaluación en los centros docentes. Madrid: MEC.

Miles, M. B. y Huberman, A. M. (1994). Qualitative data analysis. San Francisco, CA: Sage. 
Ministerio de Educación y Ciencia. (2007). Real Decreto 1393/2007, de 29 de octubre por el que se establece la ordenación de las enseñanzas universitarias oficiales. B.O.E. núm. 260, de 30 de octubre de 2007, 44037-44048.

Ministerio de Educación. (2010). Estrategia universidad 2015. Contribución de las universidades al progreso socioeconómico español. Madrid: Ministerio de Educación.

Morin, E. (1994). Introducción al pensamiento complejo. Barcelona: Gedisa.

Murillo, F. J. (2011). Hacer de la educación un ámbito basado en evidencias científicas. REICE Revista Iberoamericana sobre Calidad, Eficacia y Cambio en Educación, 9(3), 4-12.

OCDE. (2005). Le rôle crucial des enseignants: Attirer, former et retenir des enseignants de qualité. París: OCDE.

OCDE. (2018). The future of education and skills education 2030. Recuperado de https://www.oecd.org/education/2030/E2030\%20Position\%20Paper\%20(05.04.2018).pdf

Prégent, R. (2001). L'encadrement des travaux de mémoire et de thèse. Conseils pédagogiques aux directeurs de recherche. Montréal: Presses internationales Polytechnique.

Puren, C. (2001). Processus et stratégies de formation à la recherche en didactique des languescultures. ÉLA Revue de Didactologie des Langues-Cultures, 123, 293-418.

Puren, C. (2015). Théorie générale de la recherche en didactique des langues-cultures. Recuperado de https://www.christianpuren.com/mes-travaux/2015a/

Rey, O. (2006). Qu'est-ce qu'une bonne recherche en éducation? Lettre D'Information de la VST, 18, 1-8.

Rey, O. (2014). Entre laboratoire et terrain: Comment la recherche fait ses preuves en éducation. Dossier de veille de l'IFÉ, 89, 1-28.

Roy, J. A. (1989). Recherche, enseignement et enseignant. Revue des Sciences de L'Éducation, 151(1), 103-122. https://doi.org/10.7202/900620ar

Sañudo, L. (2012). El papel de las redes profesionales de investigación en un mundo globalizado. REICE. Revista Iberoamericana sobre Calidad, Eficacia y Cambio en Educación, 1O(3), 135-143.

Shavelson, R. J. y Towne, L. (2002). Scientific research on education. Washington, DC: The National Academic Press.

Thünnermann, C. (2000). Universidad y sociedad: Balance histórico y perspectivas desde Latinoamérica. Caracas: Ministerio de Educación, Cultura y Deportes.

UNESCO. (1998). Conferencia Mundial sobre la educación superior. La educación superior en el siglo XXI. Visión y acción. París: UNESCO.

Universidad de Valladolid. (2018). Trabajo fin de grado-lengua extranjera-francés. Recuperado de https://alojamientos.uva.es/guia_docente/uploads/2018/404/40550/1/Documento 1.pdf

Van der Maren, J. M. (1996). Méthodes de recherche pour l'éducation. Éducation et formation. Fondements. Bruselas: De Boeck y Larcier.

Wanlin, P. (2007). L'analyse de contenu comme méthode d'analyse qualitative d'entretiens: Une comparaison entre les traitements manuels et l'utilisation de logiciel. Recherches Qualitatives, 3, 243-272.

Whitty, G. (2006). Education(al) research and education policy making: Is conflict inevitable? British Educational Research Journal, 32(2), 159-176. https://doi.org/10.1080/01411920600568919 


\section{Breve CV de los autores}

\section{Carmen Guillén-Díaz}

Licenciada y Doctora en Filología Francesa por la Universidad de Valladolid y Premio Extraordinario de Doctorado. Profesora Titular de Universidad. Área de Didáctica de la Lengua y la Literatura. Evaluada como "Excelente" en 2011, según el Programa DOCENTIA acreditado por la ANECA. Con docencia en formación inicial y continua del profesorado de lenguas para los distintos niveles educativos; en formación a la investigación en el marco del Programa de Doctorado de Didáctica de la Lengua y la Literatura de la Universidad de Valladolid (1986-2012); en el Master Universitario en Psicopedagogía y en el Máster en Investigación Aplicada a la Educación. Línea de investigación: la profesionalización y desarrollo profesional de los docentes, concretada en la dirección de 12 tesis doctorales defendidas, en la dirección de numerosos trabajos de investigación tutelados y en libros y artículos en revistas especializadas. ORCID ID: https://orcid.org/0000-0002-3239-824X.Email: ccmc@dlyl.uva.es

\section{Francisco Javier Sanz-Trigueros}

Doctor en Ciencias de la Educación por la Universidad de Valladolid y Profesor Asociado del Departamento de Didáctica de la Lengua y la Literatura de esa Universidad. Imparte docencia en los Grados de Educación Infantil y Primaria y en el Máster en Profesor de Educación Secundaria Obligatoria y Bachillerato, Formación Profesional y Enseñanza de Idiomas. Tutela los correspondientes Trabajos Fin de Grado y Máster, y coordina el Practicum del Grado en Educación Primaria. Desarrolla su investigación en torno al desarrollo profesional de docentes de y en Lenguas extranjeras y a la construcción de procesos de cualificación, difundiéndola en publicaciones y en reuniones científicas. Es miembro del GIR Q-ESE de la Universidad citada, del Observatorio Europeo del Plurilingüismo y Responsable de Intercambio Bilateral. ORCID ID: https://orcid.org/0000-0001-5740-7002.Email: franciscojavier.sanz.trigueros@uva.es 